\title{
FAMILIAL DEAFNESS ASSOCIATED WITH IRIS DEGENERATION AND GLAUCOMA
}

\author{
PAUL RUNDLE, ANDREW J. LOTERY, DESMOND B. ARCHER and \\ F. GERARD McGINNITY \\ Belfast
}

\begin{abstract}
SUMMARY
The association between iris abnormalities and glaucoma is well established. We report a family presenting a unique iris morphology, associated with familial deafness and, in two cases, glaucoma. This condition appears to be inherited as an autosomal dominant trait.

The association of iris abnormalities and glaucoma forms part of a number of congenital syndromes including the mesodermal dysgeneses, ${ }^{1}$ aniridia ${ }^{2}$ and the irido-corneal endothelial syndromes, ${ }^{3}$ as well as degenerative conditions such as iridoschisis. ${ }^{4}$ In addition, deafness and iris heterochromia together form part of Waardenburg's syndrome. ${ }^{5}$ In this paper we describe a family suffering from an unusual iris degeneration, associated with sensorineural deafness/tinnitus and glaucoma, which appears to be inherited as an autosomal dominant trait.
\end{abstract}

\section{PATIENTS AND METHODS}

A 75-year-old man presented to the eye clinic having been noted by his optician to have raised intraocular pressure. Examination revealed bilateral peripupillary stromal atrophy giving the appearance of a double iris. There were none of the classical features of iridoschisis, iris transillumination nor posterior embryotoxon. Both anterior chambers were shallow (10 degrees, Grade 1 Shaffer grading system ${ }^{6}$ ). Gonioscopy was otherwise normal. In particular there were no iris processes, excessive pigmentation or neovascularisation. Intraocular pressures were recorded as $30 \mathrm{mmHg}$ on the right and $22 \mathrm{mmHg}$ on the left. Visual fields were full; however, there was asymmetry of the cup/disc ratio ( 0.5 right, 0.3 left). Pupils dilated poorly with guttae cyclopentolate $1 \%$

From: Department of Ophthalmology, Royal Group of Hospitals, Belfast, UK.

Correspondence to: Mr Paul Rundle, FRCOphth, Eye and Ear Clinic, Royal Victoria Hospital, Grosvenor Road, Belfast 12 6BA, Northern Ireland. and phenylephrine $10 \%$. Pupil reactions were normal. Fundoscopy revealed no evidence of a pigmentary retinopathy. Questioning revealed that the patient had a moderate hearing impairment and had suffered from tinnitus for 12 years. A fluorescein iris angiogram was performed which showed hypoperfusion of the iris radial vessels with staining of the vessel walls together with gross peripupillary leakage suggestive of neovascularisation (Fig. 1).

Examination of the family revealed a further 6 members with similar iris changes of varying degree (Fig. 2). Patient II:7 (80 years of age) had identical changes to the propositus and had previously undergone peripheral iridectomies for angle-closure glaucoma (Fig. 3). In addition she had suffered from sensorineural deafness for 30 years. This patient underwent a routine cataract extraction at which an iris biopsy was taken. This showed non-specific chronic inflammatory changes and a peripupillary neovascular membrane. Chromosomal analysis of this patient revealed a normal female karyotype. Patient II:9 (67 years) had identical iris changes and

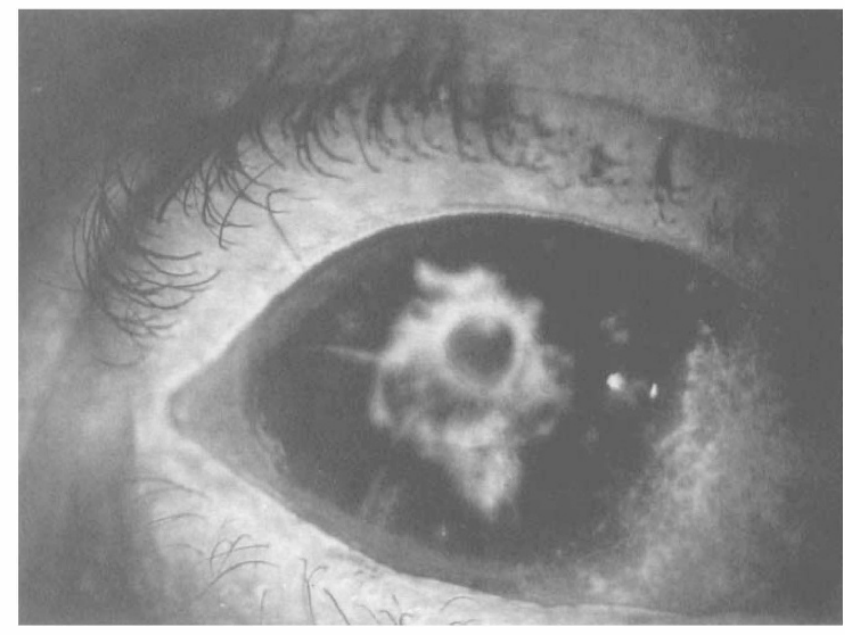

Fig. 1. Iris angiogram of the presenting patient showing hypoperfusion, vascular staining and peripupillary leakage. 

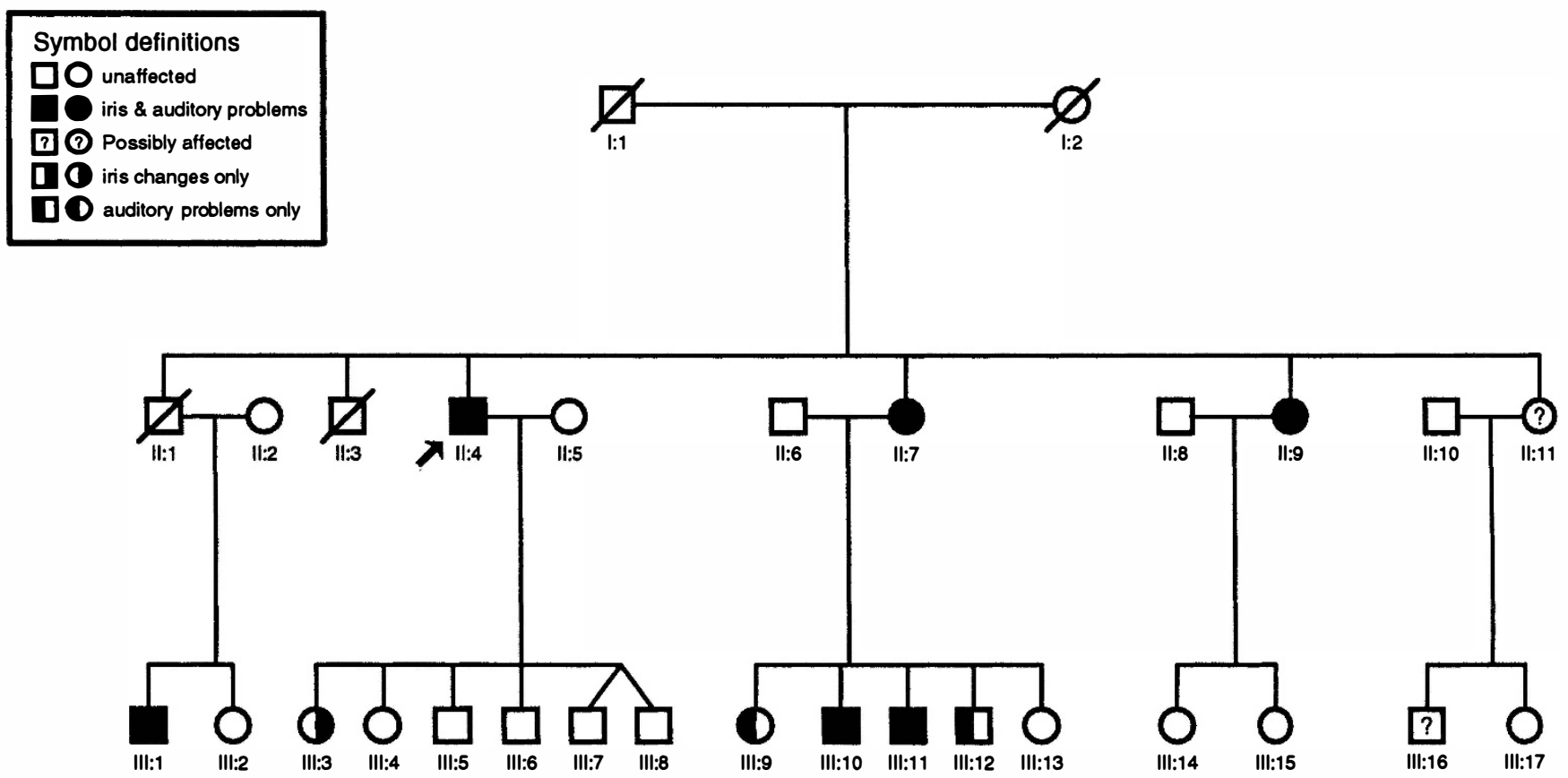

Fig. 2. Pedigree of family with iris degeneration, deafness and glaucoma. Arrow indicates proband. All patients except II:10, II:11 and III:16 were examined.

complained of hearing loss, although this had never been confirmed by audiometry. Patients III:10 (51 years) and III:11 (49 years) had similar but less extensive iris changes than their mother, associated with hypermetropia but with no evidence of glaucoma to date. These two patients together with two other siblings, III:9 and III:12, all suffered from profound sensorineural deafness from childhood. One patient, III:1 (43 years), had early iris changes associated with tinnitus. The age of those affected ranged from 43 to 80 years, the extent of the iris changes increasing with age. All the affected individuals had brown irides. There were no features suggestive of the mesodermal dysgeneses nor of aniridia. Furthermore there was no heterochromia, telecanthus nor other pigmentary abnormalities suggestive of Waardenburg's syndrome.

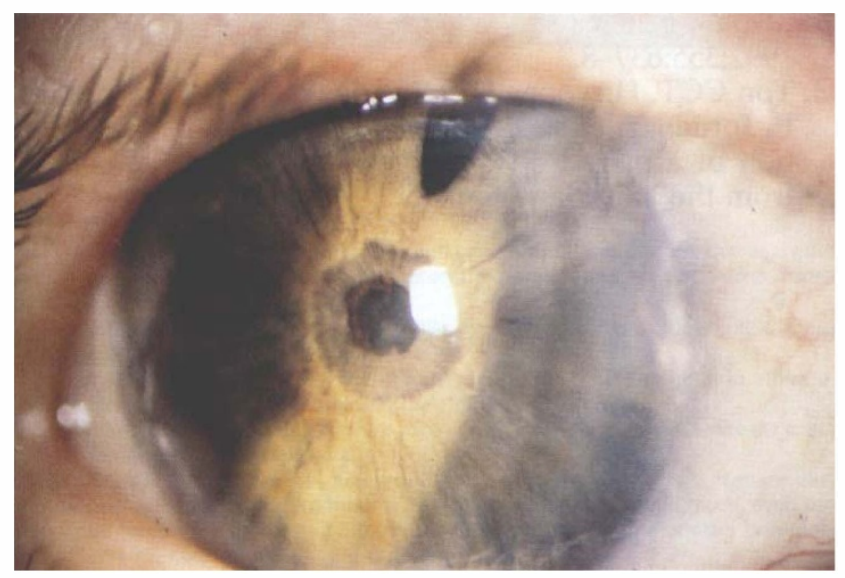

Fig. 3. Typical iris appearance showing peripupillary atrophy and peripheral iridectomy.
Unfortunately, we were not able to examine patient II:11 or her son, patient III:16. He is known to have been deaf from childhood whilst his mother defaulted from follow-up some years ago having been noted to have raised intraocular pressure ( 26 $\mathrm{mmHg}$ ).

A fluorescein angiogram performed on patient III:9 showed rapid filling of the conjunctival and scleral vessels but markedly reduced filling of the iris stromal vessels with virtually no identifiable dye in the radial vessels. There was a small amount of dye leakage from the pupil margins of both eyes.

\section{DISCUSSION}

Many syndromes have been described linking various forms of iris atrophy/degeneration with glaucoma. Iridoschisis is a rare senile atrophy of the iris stroma associated with angle-closure glaucoma in up to $50 \%$ of cases. ${ }^{7}$ Cases have also been described following trauma, ${ }^{4}$ or occurring as a senile degeneration. ${ }^{7}$ Dominant inheritance has been described previously. ${ }^{8}$ The characteristic features of iridoschisis are splitting of the iris stroma (often of the inferior iris) into fibres, the ends of which float freely in the anterior chamber, giving the iris a frayed appearance. Gonioscopy on such cases often shows shallow anterior chambers with or without anterior synechiae. ${ }^{4,7}$ Furthermore, it appears that, when associated with glaucoma, the iris changes are the result of raised intraocular pressure subsequent to the angle closure rather than the cause of the glaucoma itself. ${ }^{4}$ The iris changes evident in this family are different in that they antedate any rise in intraocular pressure. Secondly, they involve an 
apparently progressive atrophy/retraction of the anterior iris plate, as far back as the collarette in the oldest affected individuals. There was no fibrillary degeneration.

With regard to the angiographic findings in the two patients tested, previous studies of iridoschisis have shown apparently normal perfusion. Our findings of marked hypoperfusion of the iris are similar to those of Brooks and Gillies. ${ }^{9}$ They described 17 patients with iris stromal atrophy, different from that described here, who all showed sectorial hypoperfusion of the iris and peripupillary leakage of fluorescein. As patients III:9 and III:12 have similar iris colouration and deafness the question remains whether, in the future, they too will develop the iris abnormality shown by their siblings. Certainly, if iris hypoperfusion as demonstrated on angiography plays a role in the aetiology of these changes, then patient III:9 would appear to be at risk.

The mechanism underlying the deafness and ocular abnormalities in this family is unclear. Since both the iris stroma and inner ear are derived from neural crest cells, the changes may represent a progressive abiotrophic degeneration of neural crest derivatives. With regard to the iris degeneration, the combination of vascular staining and neovascularisation would suggest ischaemia as a mechanism. On the other hand, the vascular incompetence observed on angiography may merely be an epiphenomenon. It is known, for instance, that in urethane-induced retinopathy, loss of apposition between glia and retinal vessels leads to fenestration of the normally 'tight' retinal vasculature with subsequent leakage. ${ }^{10}$ It may be that the iris vascular changes evident in this family represent a similar phenomenon. Whether similar vascular abnormalities underlie the deafness is unknown.

Previous work has shown that the so-called Pax genes play an important role in the migration and differentiation of neural crest cells. ${ }^{11}$ Mutations of Pax -3 and Pax-6 are now known to be responsible for the abnormalities of neural-crest-derived tissues that form part of Waardenburg's syndrome ${ }^{12}$ and aniridia respectively. ${ }^{13}$ It may be that there is a similar basis to the abnormalities seen in the family described here. Further work is awaited regarding the genetic defect underlying this interesting syndrome.

This work was presented in part as a poster at the Oxford Ophthalmological Congress 1995. The authors would like to thank Miss G. Silvestri for helpful advice and Dr Roy Lyness for examining the pathology specimen.

Key words: Iris degeneration, Deafness, Glaucoma, Fluorescein angiography

\section{REFERENCES}

1. Gregor Z, Hitchings RA. Rieger's anomaly: a 42 year follow up. Br J Ophthalmol 1980;64:56-8.

2. Grant WM, Walton DS. Progressive changes in the angle in congenital aniridia, with development of glaucoma. Am J Ophthalmol 1974;78:842-7.

3. Eagle RC, Font RL, Yanoff M, et al. Proliferative epitheliopathy with iris abnormalities. Arch Ophthalmol 1979;97:2104-9.

4. Mills PV. Iridoschisis. Br J Ophthalmol 1967;51:158-64.

5. Waardenburg PJ. A new syndrome combining developmental anomalies of the eyelids, eyebrows and nose root with pigmentary defects of the iris, head and hair and with congenital deafness. Am J Hum Genet 1951; 3:195-253.

6. Shaffer RN. Gonioscopy, ophthalmoscopy and perimetry. Trans Am Arch Ophthalmol 1960;64:112.

7. Carter LF. Progressive senile fibrillar atrophy of the iris stroma (iridoschisis). Am J Ophthalmol 1953;36: 967-70.

8. Mansour MA. A family with iridoschisis, narrow anterior chamber angle, and presenile cataract. Ophthalmic Paediatr Genet 1986;7:145-9.

9. Brooks AMV, Gillies WE. Iris atrophy with hypoperfusion and microneovascularisation. Br J Ophthalmol 1987;71:706-15.

10. Tyler NK, Burns MS. Alteration in glial cell morphology and glial fibrillary acidic protein expression in urethane-induced retinopathy. Invest Ophthalmol Vis Sci 1991;32:246-56.

11. Hogan BLM, Horsburgh G, Cohen J, Hetherington CM, Fisher G, Lyon MF. Small eyes (Sey): a homozygous lethal mutation on chromosome 2 which affects the differentiation of both lens and nasal placodes in the mouse. J Embryol Exp Morphol 1986;97:95-110.

12. Baldwin CT, Hoth CF, Amos DA, da Silva EO, Milunsky A. An exonic mutation in the HuP2 paired domain gene causes Waardenburg's syndrome. Nature 1992;355:637-8.

13. Ton CCT, Hirvonen H, Miwa H, Well MM, Monaghan $\mathrm{P}$, Jordan $\mathrm{T}$, et al. Positional cloning and characterisation of a paired box- and homeobox-containing gene from the aniridia region. Cell 1991;67:1059-74. 